\title{
ELECTRODE GEOMETRY AND PREFERENTIAL STIMULATION OF SPINAL NERVE FIBERS HAVING DIFFERENT ORIENTATIONS : A MODELING STUDY
}

\author{
Jan Holsheimer, Johannes J. Struijk
}

Biomedical Engineering Division, Dept. of Electrical Engineering, University of Twente, Enschede, The Netherlands

\begin{abstract}
In a computer modeling study of epidural spinal cord stimulation using a longitudinal array of electrode contacts, the effect of contact geometry and contact combination on the threshold voltages for stimulation of dorsal column (DC) fibers and dorsal root (DR) fibers was investigated. It was concluded that DC-fiber stimulation will be favoured when a tripolar combination and small contact length and spacing are used, while DR-fiber stimulation will be favoured when unipolar stimulation and large contact length are used.
\end{abstract}

\section{INTRODUCTION}

From clinical experience in epidural spinal cord stimulation (ESCS) it is assumed that nerve fibers in both the dorsal columns (DC) and the dorsal roots (DR) can be stimulated, and moreover that DR-fiber stimulation is responsible for discomfort and motor effects in patients. In general only a small difference exists beween the threshold voltages for evoking paresthesia (related to therapeutic effects) and for inducing unwanted effects. DC-fibers and DR-fibers have different orientations and will thus be affected differently by the imposed electrical field [1]. Therefore, it was investigated theoretically how stimulation of DC-fibers could be selectively reinforced by varying the geometry of the longitudinal (epidural) contact array and the contact combination, in order to increase the "usage range" in ESCS and thereby its therapeutic benefit.

\section{METHODS}

The computer model consists of two parts.

1. A 3-dimensional volume conductor model, representing the gross anatomy and electrical conductivities of a spinal cord segment and surrounding anatomical structures, e.g. the cerebrospinal fluid (csf), dura, epidural fat and vertebral bone, as well as the geometry and position of the epidural lead contacts. A transverse section of the model is shown in fig. 1 . Grid spacings varied from $0.15 \mathrm{~mm}$ to $1.6 \mathrm{~mm}$, the smallest values being near the contacts and the dorsal columns. The electrical potential field in this model was calculated by solving the discretized Laplace equation by a Red-black GaussSeidel iteration, using the contacts and the boundary of the model as voltage sources (Dirichlet conditions)

The conductivity of the dura was chosen such that the tissue resistance between contacts matched the average value measured in patients when using the corresponding contact geometry in unipolar and bipolar stimula-

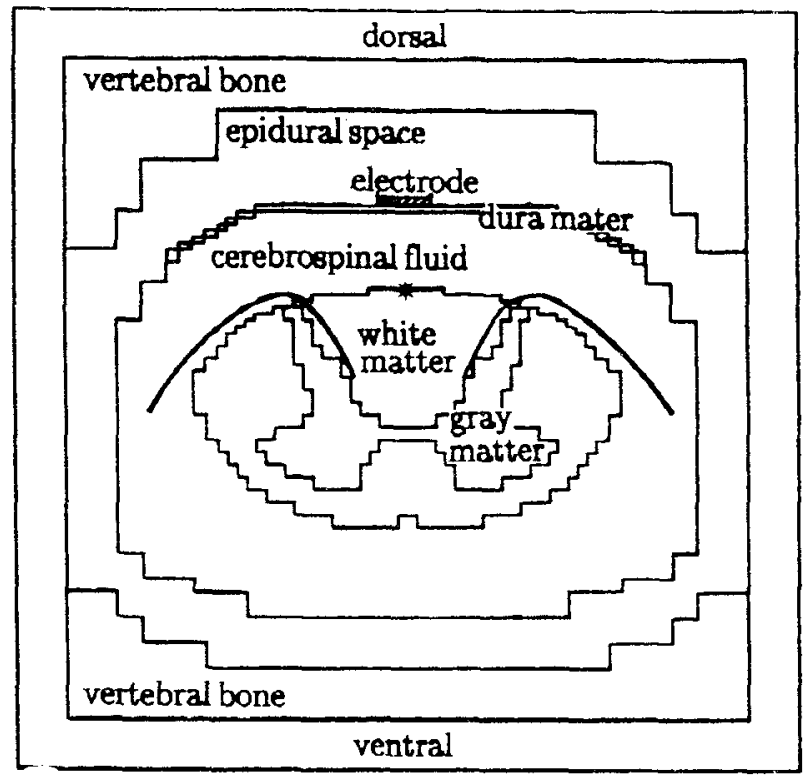

Fig. 1. Volume conductor model of cervical spinal cord: transverse section.

tion ( $1090 \Omega$ and $715 \Omega$, respectively). In the model the resistance was obtained by calculating the current at the surface of each contact.

2. A McNeal type model of myelinated nerve fiber [2], representing the electrical behavior of a DC-fiber or a DR-fiber in the calculated electrical field. Threshold voltages (between contacts) for the excitation of these fibers were calculated for a $210 \mu$ s rectangular stimulus pulse. The (cervical) DR-fiber was in a transverse plane and the DC-fiber was at the dorsomedial border of the dorsal columns, as indicated in fig. 1. Both the DR-fiber and the DC-fiber had diameters of $6 \mu \mathrm{m}$.

According to McNeal the change in nodal membrane potential of a fiber is related to the second order difference of the nodal field potentials. We have shown theoretically that a DR-fiber will be excited close to its entry into the spinal cord [3].

Three contact combinations were used: a bipolar, a tripolar (central cathode) and a unipolar one (epidural cathode, boundary of the volume conductor is the anode). Contacts in the epidural space, bordering the dura dorsomedially, had a cubic shape. Contact width was varied from $0.3 \mathrm{~mm}$ to $4.8 \mathrm{~mm}$, while both contact length and contact spacing were varied from $1.2 \mathrm{~mm}$ to $9.6 \mathrm{~mm}$. 
Because we have shown theoretically that the width of the dorsal csf-layer largely influences threshold voltages $[3,4]$, this model parameter was also varied $(1.2 \mathrm{~mm}-3.6 \mathrm{~mm})$.

\section{RESULTS}

\section{Tissue resistance}

Calculation of the tissue resistance between contacts showed that this parameter mainly depends on the contact area and the conductivity of the neighboring tissue. Therefore, we found that resistance in tripolar stimulation is lower than in bipolar stimulation. Unipolar stimulation, however, had the lowest value, because the large anodal contact is the case of the implanted stimulator (modeled by the outer boundary of the volume conductor). Due to the high conductivity of the csf the resistance only changed slightly when varying the width of the dorsal csf-layer.

\section{Contact combination}

When identical contact geometries were used, the lowest threshold voltages for both the DC-fiber and the DR-fiber were obtained with unipolar stimulation, and the highest with bipolar stimulation. A comparison of DC-fiber and DR-fiber threshold voltages (V) obtained with various contact geometries showed that the ratio VDR/VDC is lowest in unipolar stimulation and highest in tripolar stimulation.

\section{Contact width}

When contact width was increased, both VDR and VDC decreased for all three contact combinations, due to a reduction of tissue resistance.

\section{Contact length and spacing}

VDR and VDC are high for a small contact length or contact spacing. As one of these variables is increased, VDR and VDC decrease to a minimum value and then increase slightly. However, the contact lengths or spacings related to the minimum of VDC and VDR are different $(\simeq 2 \mathrm{~mm}$ and

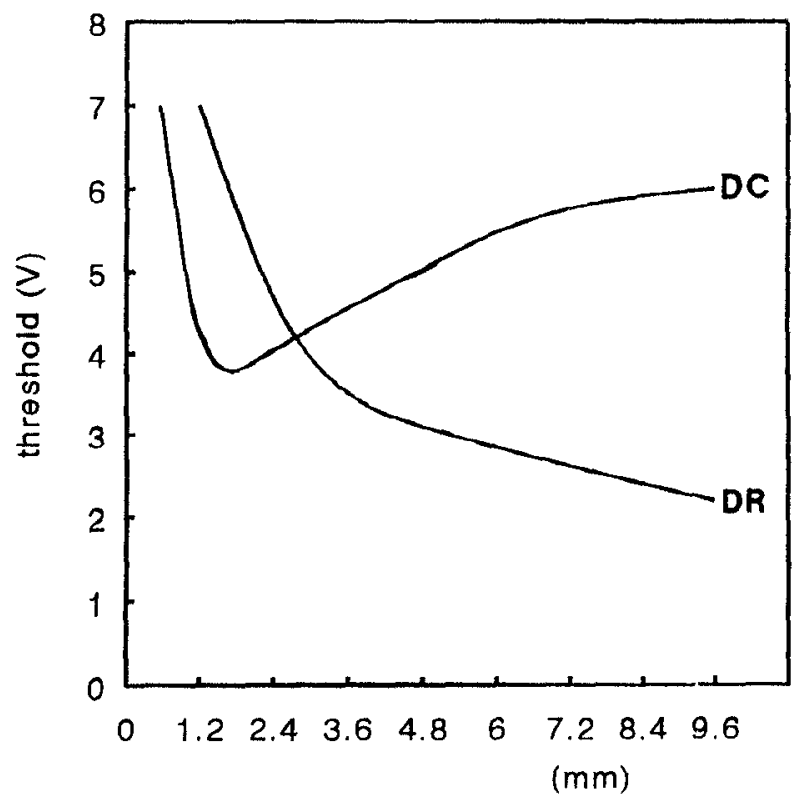

Fig. 2. DC-fiber and DR-fiber thresholds as a function of contact length in bipolar epidural stimulation (spacing = $4.8 \mathrm{~mm}$ ). $\simeq 20 \mathrm{~mm}$ in the example shown in fig. 2). Therefore, changes in contact length or contact spacing have opposite effects on VDR and VDC.

\section{Width of dorsal csf layer}

Increasing the width of the csf-layer between epidural contacts and spinal cord resulted in a strong increase of both VDR and VDC. At increasing csf thickness the ratio VDR/VDC decreased, which means an increase of DR-fiber preference. Therefore, the optimal contact geometry to obtain a small or high ratio VDR/VDC varies with csfwidth.

\section{DISCUSSION}

It was found that a slight increase in the conductivity (or thickness) of the tissue between contacts and csf-layer results in a large increase of both tissue resistance and threshold voltages. When the width of the csf-layer was increased threshold voltages also increased strongly, but tissue resistance only increased slightly. Due to random variations of both parameters among patients threshold voltage will not be correlated with tissue resistance, neither with csf-width.

The modeling study predicts that length and spacing of contacts in a logitudinal array of epidural stimulating contacts have opposite influences on the threshold voltages for the recruitment of DC-fibers and DR-fibers when these parameters are varied between 2 and $20 \mathrm{~mm}$. The ratio VDR/VDC decreases with increasing contact length or contact spacing. This means that increases in these geometric parameters reduce the gradient of the $\mathrm{E}-$-field in a longitudinal direction (DC-fibers), while increasing the gradient in a radial direction with respect to the contact array (the direction of DR-fibers at their entrance in the spinal cord).

Moreover, the ratio VDR/VDC is highest in tripolar stimulation and lowest in unipolar stimulation. Therefore, stimulation of DC-fibers or DR-fibers can be selectively reinforced by both the geometry of the contact array and the contact combination. DC-fiber stimulation will be favoured when using a tripolar combination and a small contact length and spacing, while DR-fiber stimulation will be favoured in unipolar stimulation and a large contact length. However the optimal contact geometry according to any criterion largely depends on the anatomical relations, especially the width of the dorsal csf-layer and the geometry of the spinal cord. Therefore, the optimal geometry will vary at different segmental levels. We have recently investigated these anatomical relations at various levels of the spinal cord by MRI (Turbo Scan Echo technique) on healthy subjects.

\section{REFERENCES}

[1] J.J. Struijk, J. Holsheimer, B.K. van Veen, H.B.K. Boom, "Epidural spinal cord stimulation: Calculation of field potentials with special reference to dorsal column nerve fibers," IEEE Trans. Biomed Eng., vol. 38, pp. 104-110, 1991.

[2] D.R. McNeal, "Analysis of a model for excitation of myelinated nerve," IEEE Trans. Biomed. Eng., vol. 23, pp. 329-337, 1976.

[3] J.J.Struijk, Immediate effects of spinal cord stimulation, Thesis, University of Twente, Enschede, 1992.

[4] J. Holsheimer, J.J. Struijk, "How do geometric factors influence epidural spinal cord stimulation? A quantitative analysis by computer modeling," Stereotact. Funct. Neurosurg., vol. 56, pp. $234-249,1991$ 